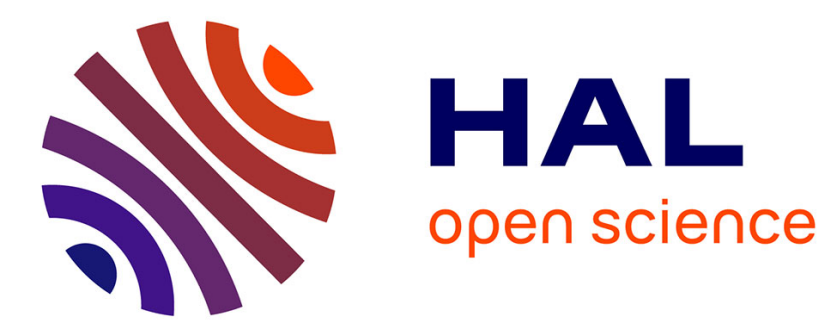

\title{
Rasterization strategies for airborne LiDAR classification using attribute profiles
}

Florent Guiotte, Sébastien Lefèvre, Thomas Corpetti

\section{To cite this version:}

Florent Guiotte, Sébastien Lefèvre, Thomas Corpetti. Rasterization strategies for airborne LiDAR classification using attribute profiles. 2019 Joint Urban Remote Sensing Event (JURSE), May 2019, Vannes, France. pp.1-4, 10.1109/JURSE.2019.8808945 . hal-02343901v2

\section{HAL Id: hal-02343901 \\ https://hal.science/hal-02343901v2}

Submitted on 8 Dec 2020

HAL is a multi-disciplinary open access archive for the deposit and dissemination of scientific research documents, whether they are published or not. The documents may come from teaching and research institutions in France or abroad, or from public or private research centers.
L'archive ouverte pluridisciplinaire HAL, est destinée au dépôt et à la diffusion de documents scientifiques de niveau recherche, publiés ou non, émanant des établissements d'enseignement et de recherche français ou étrangers, des laboratoires publics ou privés. 


\section{Rasterization strategies for airborne LiDAR classification using attribute profiles}

\author{
Florent Guiotte \\ LETG-Rennes, IRISA \\ Univ. Rennes 2 \\ Rennes, France \\ florent.guiotte@univ-rennes2.fr
}

\author{
Sébastien Lefèvre \\ IRISA \\ Univ. Bretagne Sud \\ Vannes, France \\ sebastien.lefevre@irisa.fr
}

\author{
Thomas Corpetti \\ LETG-Rennes \\ CNRS \\ Rennes, France \\ thomas.corpetti@univ-rennes2.fr
}

\begin{abstract}
This paper evaluates rasterization strategies and the benefit of hierarchical representations, in particular attribute profiles, to classify urban scenes issued from multispectral LiDAR acquisitions. In recent years it has been found that rasterized LiDAR provides a reliable source of information on its own or for fusion with multispectral/hyperspectral imagery. However previous works using attribute profiles on LiDAR rely on elevation data only. Our approach focuses on several LiDAR features rasterized with multilevel description to produce precise land cover maps over urban areas. Our experimental results obtained with LiDAR data from university of Houston indicate good classification results for alternative rasters and even more when multilevel image descriptions are used.
\end{abstract}

Index Terms-airborne LiDAR, land cover mapping, attribute profiles, multilevel image description

\section{INTRODUCTION}

Airborne LiDAR systems are a common source of aquisition for elevation data. Such systems provide accurate 3D point clouds of the scanned scenery. LiDAR is very popular over urban areas where it brings a valuable complementary source of information when used with multispectral or hyperspectral optical data in order to achieve land cover or land use mapping.

LiDAR data are voluminous, irregularly distributed point clouds coming along with intensity features and acquisition meta-data. Due to this complexity, LiDAR data for land cover mapping are often simplified to a digital elevation model (DEM) used as additional information for fusion with multispectral or hyperspectral images. In this work, we focused on providing such maps with LiDAR data only using multilevel image description. Classification of several urban classes was derived based on features from LiDAR data such as intensities, elevation and number of echoes.

In the following we review simple yet effective rasterization strategies of LiDAR data, that are subsequently used for multilevel image description with attribute profiles (APs). By doing so, we are then able to efficiently derive a precise land cover map through supervised classification.

The authors acknowledge the support of Région Bretagne (CAMLOT doctoral project).

\section{RELATED WORK}

\section{A. Classification of LiDAR data}

Numerous methods have already been proposed in the past decade for LiDAR point cloud classification, coming from various scientific fields such as geosciences (flow, erosion, rock deformations, ...), computer graphics (3D reconstruction) or Earth observation (detection of trees, roads, buildings, ...).

Among efficient techniques, some directly exploit the 3D point cloud structure [1], [11], [13] while in many applications the point cloud is first binned into a 2D regular grid (rasterization process) on which computer vision approaches can be applied (see e.g. [10]). Apart from some specific applications where LiDAR points are fused with other data (e.g. hyperspectral images [6]-[8], [14]), most techniques consist in computing features to describe the point clouds, before using such features to classify the scene under study. While first works have been focused on the characterization of single points (often through height and intensity) without including information related to their neighbours [10], more advanced approaches have included spatial relationships using a set of spheres or cylinders (of variable radius) around each point to extract consistent geometric features [11], [13], [16]. In this context, multiscale local 3D features (main orientation, variability around each point, ...) have proven their efficiency to classify LiDAR scenes (see e.g. [1]). Even if it is very efficient, the sphere used to assess the neighbourhood of points is isotropic (no orientation is promoted) which is not optimal since the geometry of objects is not taken into account. Therefore other multi-scale approaches have been proposed on LiDAR DEM, such as the popular attribute profiles [5] that produce a multiscale description of the pixel and its surrounding [7], [8], [14] before proceeding to the classification. The main idea behind is to compute multi-scale spatial features by taking into account the geometry of the scene. In this work, we suggest to explore various information derived from the LiDAR point cloud within the framework of attribute profiles.

\section{B. Attributes profiles}

Introduced in 2010 for remotely-sensed images, morphological APs [5] enable a multi-scale description of data driven by their spatial and spectral information. Efficient computation 


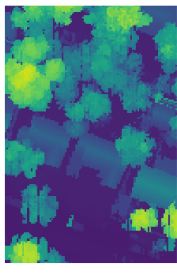

(a) First echo

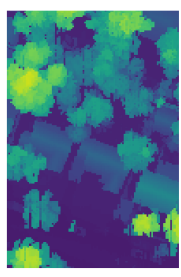

(b) $\Lambda=10$

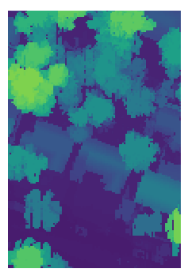

(c) $\Lambda=200$

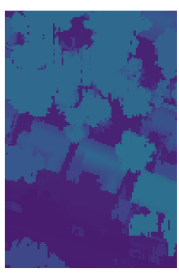

(d) $\Lambda=5000$

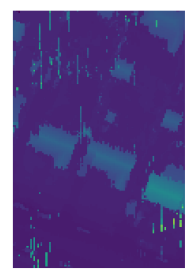

(e) Last echo

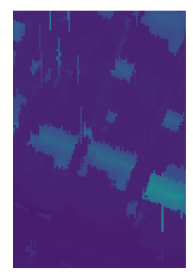

(f) $\Lambda=10$

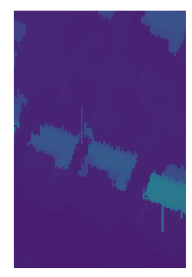

(g) $\Lambda=200$

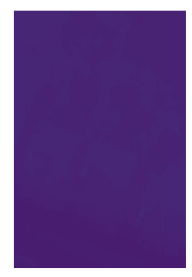

(h) $\Lambda=5000$

Fig. 1: Some rasters over residential area, for first echo (a-b-c-d) and last echo (e-f-g-h) with original rasters (a-e), SDAP filtering with $\Lambda=10$ (b-f), $\Lambda=200$ (c-g) and $\Lambda=5000$ (d-h). One can observe the interest of the last echo (second line) since structures below vegetation are clearly highlighted.

of APs is achieved through tree-based representation of the gray level sets with either a max or a min-tree. They have then been superseded by self-dual attribute profiles (SDAPs) [4] built from a unique multi-scale representation of an image through the tree of shapes. In this tree, all nodes represent nested connected regions of similar pixels, with leaves made of the local extrema and the root gathering all pixels of the image. Then, successive filterings of the image (or equivalently the tree representation) are performed according to some predefined characteristics computed for each node such as area, moment of inertia, or standard deviation of the connected components. The filtered images are finally stacked together to form description vectors called SDAPs. The concatenation of the SDAPs from different bands in a single vector are called extended self-dual attribute profiles (ESDAPs) [2]. From the concept of SDAPs, the differential self-dual attribute profiles (DSDAPs) contains the same information but expresses the difference between successive levels of the SDAPs.

Previous works combining APs and LiDAR data only focus on DEM (cf. Sec. II-A). Yet, in recent LiDAR acquisition systems, multi-spectral information can be extracted and the question of the description of other features than DEM is open.

In this study we aim to enhance existing LiDAR classification methods using SDAPs and their derivative to better describe several features extracted from LiDAR data. Validation is performed on the IEEE DFC 2018 public dataset.

\section{RASTERIZATION STRATEGIES}

In this section we provide the features we extracted from LiDAR data and the multi-scale filtering we have chosen for the spatial description.

\section{A. LiDAR features}

LiDAR systems are usually exploited to provide unstructured 3D point clouds used to derive a DEM. Though a DEM brings useful information, additional features issued from LiDAR can still be exploited, in particular:

1) The spectral intensity associated with the first echo in each spectral band.

2) The number of echoes in each spectral band. Some structures, especially in vegetated areas, do not fully backscatter the laser pulse and yield in multi-echoes signals.
3) The position of the last echo. For multi-echoes backscattered signals, this enables to localize the last element encountered. In some situations (especially with trees), this enables to localize the ground surface.

4) The associated intensities of last echo. Analogously to the paired information of first echo position and intensities we have used the last echo spectral intensities in addition to its position.

The two latter types of information are usually less employed. Nevertheless, they can help the classification process since all wooded areas are removed, as illustrated by Fig. 1(e).

The rasterization process aims to provide in each cell some representative values of the aforementioned features. For the sake of simplicity, we have chosen to average the values of intensity, elevation and number of echoes contained in each cell. Furthermore, we fill potential empty cells (missing data) through linear interpolation.

\section{B. Attribute filtering}

As previously indicated, we consider here the application of attribute profiles over different rasterized versions of LiDAR data. In this paper, for the sake of simplicity, we have chosen to filter all LiDAR features solely based on the area attribute whose values have been set in the urban context. More precisely, we consider three main scales (in other contexts, automatic approaches can be used [3]):

- small values (1 to $5 \mathrm{~m}^{2}$ ) remove small-sized objects (e.g. power lines) and can be regarded as denoising filters;

- moderate values (5 to $50 \mathrm{~m}^{2}$ ) remove medium-sized objects (cars, trees, ....);

- large values (more than $50 \mathrm{~m}^{2}$ ) remove larger objects (e.g. buildings) and therefore enable to automatically derive a digital terrain model (DTM).

\section{EXPERIMENTS}

\section{A. Dataset and setup}

1) Dataset: Our method was tested on the multi-spectral LiDAR acquisition of the University of Houston issued from 2018 IEEE GRSS Data Fusion Contest dataset ${ }^{1}$. The associated ground truth map has a spatial resolution of $0.5 \mathrm{~m}$.

\footnotetext{
${ }^{1} \mathrm{cf}$ http://www.grss-ieee.org/community/technical-committees/data-fusion/ 2018-ieee-grss-data-fusion-contest/
} 


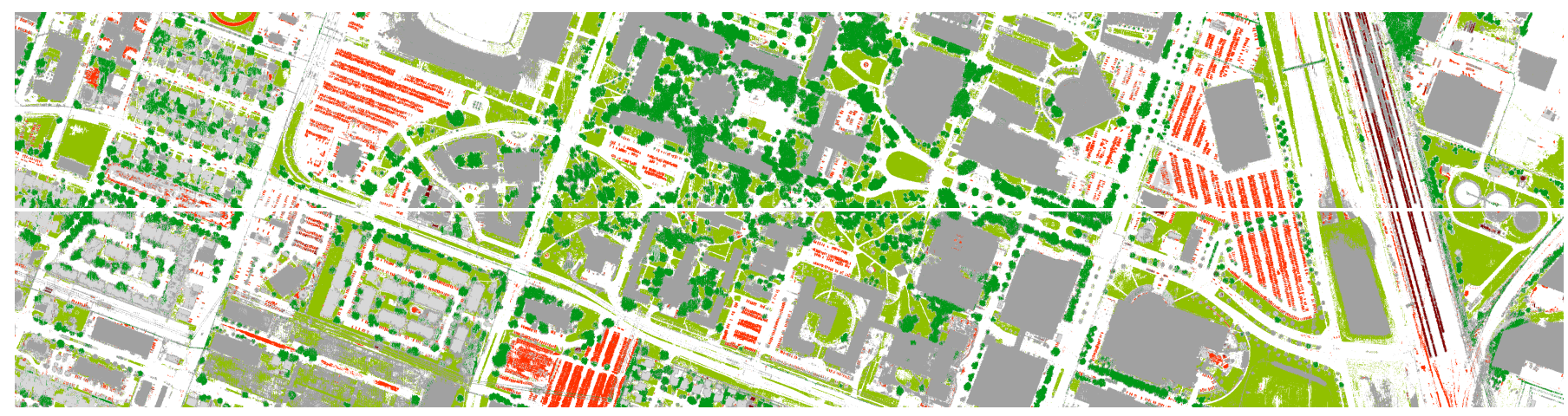

Fig. 2: Classification of the scene using DSDAPs with all features $\left\{N, I, I_{r}, D, D_{r}\right\}$. The 7 classes are represented as follows: roads in white, grass in green, trees in dark green, residential buildings in light grey, non-residential buildings in medium grey, cars in red and trains in purple.

2) Classes: We choose generic urban classes (roads, grass, trees, residential buildings, non-residential buildings, cars and trains) of the dataset to evaluate the overall accuracy.

3) Classifier: We choose random forest (RF) to classify our data since such method provides reliable results with respect both to accuracy and computational efficiency [9].

4) Feature computation: ESDAPs have been created by filtering each raster with area attributes. According to observations made in Sec. III-B, we have chosen thresholds $\Lambda=$ $\{10,200,5000\}$ to compute attributes. In practice, all features mentioned in previous sections are tested independently and combined together. These features are digital surface model (DSM) (denoted as $D$ ), intensities (denoted as $I$ ), intensities of last echo (denoted as $I_{r}$ ), number of backscattered echoes (denoted as $N$ ) and position of the last echo (denoted as $D_{r}$ ) . For the sake of comparison, we also ran the classification only with initial rasters (without multiscale analysis through SDAPs) to evaluate the benefits of APs and variants. The ESDAPs can be formulated as:

$$
E S D A P=\{S D A P(D), S D A P(I), \ldots, S D A P(N)\}
$$

5) Train and test data: Unlike most common multi-scale features that rely on spatial windows, attribute profiles rely on specific connectivity that prevents the use of random points to train and test a classifier. As a matter of fact, two pixels in various spatial areas of the image but with similar characteristics are likely to share common nodes, and hence common features. Therefore a random choice of train and test points is unfair. To cope with such a bias, one solution is to spatially split the dataset in two images of same size where the first one is used to train the classifier and the other to test it, and conversely. In practice, we split horizontally the dataset in order to maximize the class distribution in each sub-image.

6) Validation criteria: From the training split, 10\% of the points have been randomly selected to train and evaluate our approach. The process has been repeated 100 times and we provide averaged overall accuracy (OA) and Cohen's kappa coefficient $(\kappa)$ for each experience.

\section{B. Results}

Quantitative evaluations are depicted in Tab. I. As expected and reported in previous works [6], [7], [14], SDAPs improves the classification when used with DEM $D$ only (first line). This observation is almost valid for all other features. Performances of DSDAPs are slighlty higher than SDAPs except for DEM.

On this dataset, it is surprising to note that multi-spectral intensities (first echo $I$ or last echo $I_{r}$ ) are performing better than DSM $D$ (lines 5,3,1 respectively). This information is likely to be an interesting feature to separate urban elements.

It is also worth noting that regarding positions, the last echo $D_{r}$ feature is more useful than first echo $D$ (lines 1,4 ) when combined with hierarchical features, with a significant improvement (about $\sim 10 \%$ ). The ability of the last echo to assess buildings can explain such an observation.

Finally, as expected, the combination of all features (last lines) enables to achieve the best accuracy. Though the difference between DSDAPs and SDAPs is limited, the improvement with respect to the baseline is important and this demonstrates the ability of APs-based features to properly classify LiDAR data in urban environments. We can assess an improvement of $\kappa=0.06$ while comparing pixelwise and DSDAPs description both with all features. We also can assess an improvement of $\kappa=0.30$ while comparing SDAPs on DSM only with SDAPs on all LiDAR features.

For the sake of illustration, we provide in Fig. 2 the classification obtained using all features with DDSDAPs, line 14 of Tab. I. Let us remind that in practice, the complete image has been horizontally split to avoid common features issued from APs between train and test data. Therefore, as some classes appear in few parts of the image, they have not been learned and hence, not been properly classified (for instance the grassy slope at the bottom right has been classified as a building). Despite this difficulty, both quantitative evaluations and qualitative maps are interesting. The prediction map is indeed consistent as the objects exhibit few noise and proper borders. Even if some irregularities on the edges of some objects (e.g. buildings) appear, they are mainly due to the irregularity of the initial point cloud sampling. 


\begin{tabular}{|c|c|c|c|c|c|c|c|c|c|c|}
\hline & \multirow{2}{*}{$\begin{array}{l}\text { Description } \\
\text { Evaluation metric }\end{array}$} & \multicolumn{3}{|c|}{ Pixel } & \multicolumn{3}{|c|}{ E.SDAPs } & \multicolumn{3}{|c|}{ E.DSDAPs } \\
\hline & & $\mathrm{OA}(\%)$ & $\kappa(\times 100)$ & $d$ & $\mathrm{OA}(\%)$ & $\kappa(\times 100)$ & $d$ & $\mathrm{OA}(\%)$ & $\kappa(\times 100)$ & $d$ \\
\hline 1 & $D$ & 64.79 & 46.52 & 1 & 72.67 & 58.80 & 4 & 69.46 & 54.77 & 4 \\
\hline 2 & $N$ & 52.30 & 8.43 & 3 & 52.27 & 10.01 & 12 & 52.74 & 10.64 & 12 \\
\hline 3 & $I_{r}$ & 67.82 & 51.06 & 3 & 70.42 & 54.35 & 12 & 72.54 & 57.84 & 12 \\
\hline 4 & $D_{r}$ & 63.91 & 45.12 & 1 & 73.94 & 59.73 & 4 & 72.76 & 58.29 & 4 \\
\hline 5 & $I$ & 68.36 & 52.18 & 3 & 72.00 & 57.30 & 12 & 73.28 & 59.25 & 12 \\
\hline 6 & $\left\{D, D_{r}\right\}$ & 69.65 & 54.19 & 2 & 77.22 & 64.85 & 8 & 77.24 & 65.36 & 8 \\
\hline 7 & $\left\{N, D_{r}\right\}$ & 68.64 & 52.56 & 4 & 78.88 & 67.44 & 16 & 78.45 & 67.05 & 16 \\
\hline 8 & $\{I, D\}$ & 83.97 & 75.64 & 4 & 86.29 & 78.90 & 16 & 87.39 & 80.71 & 16 \\
\hline 9 & $\left\{I, D_{r}\right\}$ & 82.26 & 72.84 & 4 & 86.97 & 79.86 & 16 & 87.93 & 81.46 & 16 \\
\hline 10 & $\{N, I, D\}$ & 85.99 & 78.64 & 7 & 87.45 & 80.68 & 28 & 88.69 & 82.69 & 28 \\
\hline 11 & $\left\{N, I, D_{r}\right\}$ & 84.80 & 76.70 & 7 & 88.29 & 81.91 & 28 & 88.99 & 83.09 & 28 \\
\hline 12 & $\left\{I, D, D_{r}\right\}$ & 84.59 & 76.50 & 5 & 88.21 & 81.80 & 20 & 89.28 & 83.56 & 20 \\
\hline 13 & $\left\{N, I, D, D_{r}\right\}$ & 85.87 & 78.46 & 8 & 88.92 & 82.90 & 32 & 90.04 & 84.74 & 32 \\
\hline 14 & $\left\{N, I, I_{r}, D, D_{r}\right\}$ & 86.20 & 78.93 & 11 & 89.05 & 83.09 & 44 & 90.16 & 84.91 & 44 \\
\hline
\end{tabular}

TABLE I: Overall accuracy (OA) and Cohen's Kappa coefficient $(\kappa)$ for each feature and combination of them using pixels values, SDAPs, DSDAPs (lines 1-4) and their extended versions (lines 5-14). The dimension of the feature vector (d) is also indicated (intensities $\left(I, I_{r}\right)$ and number of echos $N$ are taken in each of the three spectral bands).

\section{CONCLUSION}

In this study, we have addressed the classification of multispectral LiDAR using rasterized features and attribute filtering. Results showed that combination of different rasterized strategies can improve classification with the sole use of LiDAR data. In addition, considering SDAPs to model multiscale spatial organization futher improved our results to the point it allowed us to produce a precise land cover map over urban area. Futhermore, the proposed method is fast (for instance, the map from Fig. 2 (1202x4768 pixels) required only 2 seconds for DSDAPs description and 50 seconds for RF classification, considering a laptop CPU (i7-7600U CPU @ $2.80 \mathrm{GHz}, 4$ threads)) and can be used with any supervised classifier (see [12]). Additional improvements can be designed for this method in the future. On the one hand, we can extract many more features from LiDAR such as point density, orientation within a cell, ratio between spectral bands and summarize the cell with other metrics than mean value such as standard deviation or quantiles. On the other hand, we can enhance SDAPs by filtering more attributes such as moment of inertia, by using more advanced APs-based methods such as the local feature-based attribute profiles (LFAPs) [15] or even by creating LiDAR specific attributes to be included during the construction of the tree. Regarding classification of overlapping classes (e.g. buildings beneath trees), it could also be interesting to head for 3D classification. With this in mind, 3D ground-truth would be optimal for LiDAR data.

\section{ACKNOWLEDGMENT}

The authors thank the National Center for Airborne Laser Mapping and the Hyperspectral Image Analysis Laboratory in University of Houston for data used in this study, and the IEEE GRSS Image Analysis and Data Fusion Technical Committee.

\section{REFERENCES}

[1] N. Brodu and D. Lague. 3d terrestrial lidar data classification of complex natural scenes using a multi-scale dimensionality criterion: Applications in geomorphology. ISPRS J. of Phot. and Rem. Sen., 68:121-134, 2012.
[2] G. Cavallaro, M. Dalla Mura, J. A. Benediktsson, and L. Bruzzone. Extended self-dual attribute profiles for the classification of hyperspectral images. IEEE Geos. and Rem. Sens. Let., 12(8):1690-1694, 2015.

[3] G. Cavallaro, N. Falco, M. Dalla Mura, and J. A. Benediktsson. Automatic Attribute Profiles. IEEE Trans. on Im. Proc., 26(4):1859$1872,2017$.

[4] M. Dalla Mura, J. A. Benediktsson, and L. Bruzzone. Self-dual attribute profiles for the analysis of remote sensing images. In Int. Symp. on Math. Morpho. and App. to Sig. and Im. Proc., pages 320-330. Springer, 2011.

[5] M. Dalla Mura, J. A. Benediktsson, B. Waske, and L. Bruzzone. Morphological attribute profiles for the analysis of very high resolution images. IEEE Trans. on Geos. and Rem. Sens., 48(10):3747-3762, 2010.

[6] B. B. Damodaran, J. Höhle, and S. Lefèvre. Attribute Profiles on Derived Features for Urban Land Cover Classification. Photo. Engineering \& Rem. Sens., 83(3):183-193, 2017.

[7] P. Ghamisi, J. A. Benediktsson, and S. Phinn. Land-cover classification using both hyperspectral and LiDAR data. International J. of Image and Data Fusion, 6(3):189-215, 2015.

[8] M. Khodadadzadeh, J. Li, S. Prasad, and A. Plaza. Fusion of Hyperspectral and LiDAR Remote Sensing Data Using Multiple Feature Learning. IEEE J. of Selected Topics in Applied Earth Observations and Remote Sensing, 8(6):2971-2983, 2015.

[9] A. Liaw, M. Wiener, and others. Classification and regression by randomForest. $R$ news, 2(3):18-22, 2002.

[10] S. K. Lodha, E. J. Kreps, D. P. Helmbold, and D. Fitzpatrick. Aerial LiDAR Data Classification Using Support Vector Machines (SVM). In Third Int. Symp. on 3D Data Proc., Vis., and Trans. (3DPVT'06), pages 567-574, Chapel Hill, NC, USA, 2006. IEEE.

[11] C. Mallet, F. Bretar, M. Roux, U. Soergel, and C. Heipke. Relevance assessment of full-waveform lidar data for urban area classification. ISPRS J. of Photogrammetry and Remote Sensing, 66(6):S71-S84, 2011.

[12] F. Merciol, T. Balem, and S. Lefèvre. Efficient and large-scale land cover classification using multiscale image analysis. In ESA Conference on Big Data from Space (BiDS), 2017.

[13] J. Niemeyer, F. Rottensteiner, and U. Soergel. Contextual classification of lidar data and building object detection in urban areas. ISPRS J. of Photogrammetry and Remote Sensing, 87:152-165, 2014.

[14] M. Pedergnana, P. R. Marpu, M. Dalla Mura, J. A. Benediktsson, and L. Bruzzone. Classification of Remote Sensing Optical and LiDAR Data Using Extended Attribute Profiles. IEEE J. of Selected Topics in Signal Processing, 6(7):856-865, 2012.

[15] M.-T. Pham, S. Lefevre, and E. Aptoula. Local Feature-Based Attribute Profiles for Optical Remote Sensing Image Classification. IEEE Transactions on Geoscience and Remote Sensing, 56(2):1199-1212, 2018.

[16] M. Weinmann, B. Jutzi, S. Hinz, and C. Mallet. Semantic point cloud interpretation based on optimal neighborhoods, relevant features and efficient classifiers. ISPRS J. of Photogrammetry and Remote Sensing, 105:286-304, 2015. 